\title{
A comparison of the effectiveness anti-platelet drugs in patients undergoing coronary artery by-pass: A clinical trial
}

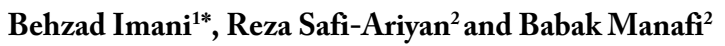 \\ ${ }^{1}$ Department of Operating room, School of Paramedicine, Hamadan University of Medical Sciences, Hamadan, Iran \\ ${ }^{2}$ Department of Cardiology, Faculty of Medicine, Hamedan University of Medical Sciences, Iran
}

\section{Background}

Platelets play a key role in cardiovascular disease both in the pathogenesis of atherosclerosis and in the development of acute thrombotic events. Their importance in vascular disease is indirectly confirmed by the benefit of antiplatelet agents in these disorders. Antiplatelet agents are the cornerstone of treatment for patients with acute coronary syndrome (ACS) undergoing percutaneous coronary intervention (PCI). Clopidogrel, has proved considerable success at reducing thrombotic complications of ACS and/or PCI compared to aspirin alone. Despite clopidogrel's ability to effectively prevent myocardial infarction (MI), stroke or death in patients with ACS and those undergoing PCI, it has limitations that compromise its clinical utility delayed onset of action, modest platelet inhibition and variability in patients' responses. Anti-platelet therapy with clopidogrel has become the standard treatment for the reduction of ischemic events in patients with coronary artery disease (CAD) with stenting, especially those undergoing drug-eluting stent (DES) implantation. Anticoagulant effect of clopidogrel is of utmost importance in coronary artery disease, especially in prevention of coronary stent thrombosis. Recently, a new local brand of clopidogrel has been launched, as Osvix ${ }^{\oplus}$ (by OSVEH Company, Tehran, Iran). This study was conducted with the aim to compare two locally prepared clopidogrel brands (Osvix ${ }^{\infty} \&$ Plavix $^{\oplus}$ ), in terms of the effect on inhibition of platelet aggregation in patients with coronary artery disease [1-6].

\section{Methods and materials}

This clinical trial study was conducted in the Cardiology department of Ekbatan Hospital (Hamadan University affiliated hospital) from June 20, 2012 to February 18, 2013. Eighty (80) patients, who were diagnosed as having coronary artery disease participated in this study, informed consent was taken from all patients and was approved by ethics committee of Hamadan University of Medical Sciences (Iran). Patients with a prior event of acute coronary syndrome, hepatic insufficiency, history of significant bleeding disorder, abnormal platelet count and those already taking anti-platelet and/or anticoagulant therapy, were excluded. The inclusion criteria for patients to study: 1 . Stenting with a stent of diameter $2.5 \mathrm{~mm}$. 2. The lack of evidence of thrombosis or dissection in the vessel prior to stenting angiographic necessary cooperation for the follow-up. The exclusion criteria for patients to study: 1. Earlier use of Ticlopidine, Plavix, Warfarin or Esophagus for any reason. 2. There is a disease with a predictable life expectancy of less than six months; 3 . Patients with chronic diseases other than cardiovascular disease (including chronic kidney, liver, respiratory, digestive, skin, etc.). The participants were randomly divided to two groups of 40 patients (by a random number generator program in MATLAB software. The research was carried out as a double-blind study and no patients or clinicians were aware of the types of prescribed drugs. To evaluate the performance of these two platelet-inhibiting drug regimens, peripheral venous blood samples were taken from patients. In order to implement the Ex-vivo analysis, blood was mixed with $3.8 \%$ citrate and platelet aggregometry was conducted. The aggregometry test was performed at $37^{\circ} \mathrm{C}$ by Iranian Blood Transfusion Organization (Tehran-Iran). The amount of Adenosine diphosphate (ADP), platelet-rich plasma and platelet count is measured as indicating factors of aggregation. The equipment used for platelet aggregation test was aggregometer, reagent, curettes, stir bars, micro-pipettes, isotonic saline, vacutte tubes and blood collecting adaptor. After collection, the blood tubes were gently inverted several times to ensure complete mixing with sodium citrate anticoagulant present in the vacutte tube. Patients' compliance was positive if they had been able to complete the bowel preparation regimen. The taste and palatability were graded as desirable and undesirable. Patient acceptance was positive if they would like to utilize the same regimen again.

\section{Results}

In total, 80 outpatients were participated in this study and divided into two groups. The patients' demographic characteristics with regard to age, gender and weight significant differences in any of these variables were observed between the two groups. The statistical analysis (by student t-test) revealed significant differences in mean of plateletrich plasma, Adenosine diphosphate and platelet count amount for both Plavix and Osvix therapeutic group $(p=0.000)$. The results show that the mean scores of well-being index for the Plavix treatment group was higher than the Osvix one. Although the Independent Sample T-test statistical analysis did not show any significant difference for both groups. Since the considered score for severe adverse side effects was 0 and the scores for moderate and mild adverse effect was 1 and 2 respectively, and 3 for non-occurrence of adverse side effects. The mean scores of adverse events for the Plavix treatment group were

${ }^{*}$ Correspondence to: Behzad Imani, Operating room Department, Paramedical School, Hamadan University of Medical Sciences, Mahdieh St, Hamadan, Iran, Tel: +988118381043, E-mail: b.imani@umsha.ac.ir

Received: May 02, 2018; Accepted: June 18, 2018; Published: June 22, 2018 
slightly higher than the Osvix one. But the Independent Sample T-test statistical analysis did not show any significant difference for both groups $(P=0.395)$. Summarize that the mean value for PRP was 236260 and $1563290 \mathrm{plt} / \mu \mathrm{l}$ for Osvix and Plavix treatment groups respectively. The ADP amount for Osvix and Plavix Group was 64.9 and $52.5 \mu \mathrm{mol}$ per $\mu$ lit and the placket count for two above mentioned groups was 186190 and 121480 per $\mu$ lit, respectively. The statistical analysis showed significant differences on Anticoagulant efficacy of Osvix and Plavix tablets.

\section{Conclusion}

The two brands of clopidogrel (Osvix \& Plavix") had a significant difference in their effect on inhibition of platelet aggregation and the Plavix brand had the higher efficacy. Some more comprehensive clinical trials recommend. There is a significant difference between the efficacy of two different brands of clopidogrel (Plavix and Osvix) on inhibition of platelet aggregation. We conclude that the effectiveness of the two different brands of clopidogrel may not be the same and this calls for further research before these preparations are used in patients with acute coronary syndrome, and after percutaneous coronary interventions. Our study supports the idea that the efficacy of a drug marketed in different brands, may not be the same. In this study, the two different brands of clopidogrel showed no significant differences on major adverse cardiac events. However, additional large-scaled, prospective randomized studies are needed to conclude that the original clopidogrel Plavix could be replaced by the generic clopidogrel Osvix:

\section{References}

1. Zahno A, Brecht K, Bodmer M, Bur D, Tsakiris DA, et al. (2010) Effects of drug interactions on biotransformation and antiplatelet effect of clopidogrel in vitro. $\mathrm{BrJ}$ Pharmacol 161: 393-404. [Crossref]

2. Suh JW, Koo BK, Zhang SY, Park KW, Cho JY, et al (2006) Increased risk of atherothrombotic events associated with cytochrome P450 3A5 polymorphism in patients taking clopidogrel. CMAJ 174: 1715-22. [Crossref]

3. Jakubowski JA, Payne CD, Li YG, Brandt JT, Small DS, et al. (2008) The use of the VerifyNow P2Y12 point-of-care device to monitor platelet function across a range of P2Y12 inhibition levels following prasugrel and clopidogrel administration. Thromb Haemost 99: 409-415. [Crossref]

4. Geisler T, Schaeffeler E, Dippon J, Winter S, Buse V, et al. (2008) CYP2C19 and nongenetic factors predict poor responsiveness to clopidogrel loading dose after coronary stent implantation. Pharmacogenomics 9: 1251-59. [Crossref]

5. Yusuf S, Zhao F, Mehta SR, Chrolavicius S, Tognoni G, et al. (2001) Effects of clopidogrel in addition to aspirin in patients with acute coronary syndromes without ST-segment elevation. $N$ Engl J Med 345: 494-502. [Crossref]

6. Fatemi MJ, S Forootan K, S Jalali SZ, Mousavi SJ, Pedram MS (2012) The effect of enoxaparin and clopidogrel on survival of random skin flap in rat animal model. World J Plast Surg 1: 64-70. [Crossref]

Copyright: (C2018 Imani B. This is an open-access article distributed under the terms of the Creative Commons Attribution License, which permits unrestricted use, distribution, and reproduction in any medium, provided the original author and source are credited. 